\title{
Advances in Antithrombotic Treatment of Coronary Heart Disease Complicated with Atrial Fibrillation
}

\author{
Zengming Qi \\ Second Affiliated Hospitial of Qiqihar Medical School; Heilongjiang Qiqihar 161006, China
}

Keywords: Coronary Heart Disease; Atrial Fibrillation; antithrombotic

\begin{abstract}
Atrial fibrillation (AF) is one of the most common clinical arrhythmias and its incidence increases with age. There are approximately 33.5 million cases of atrial fibrillation globally, and it is expected that the number of patients suffering from 2060 will increase by a factor of 2 . There are many common risk factors for atrial fibrillation and coronary heart disease, such as age, hypertension, and diabetes. In clinical practice, the two diseases often coexist, and about one-third of patients with atrial fibrillation have coronary heart disease. Atrial thrombosis and coronary thrombosis are different mechanisms, and the two antithrombotic treatment strategies are also different. Antiplatelet drug therapy is the basis and key to secondary prevention of coronary heart disease, and atrial fibrillation relies on oral anticoagulation drugs to reduce the risk of stroke and other thromboembolic events. The difficulty in the treatment of coronary heart disease with atrial fibrillation is that antiplatelet drugs and anticoagulant drugs cannot be completely replaced, and the combined use faces an increased risk of bleeding. Therefore, how to minimize the risk of bleeding while maximizing benefit is the key to the development of coronary heart disease with atrial fibrillation antithrombotic treatment.
\end{abstract}

\section{Introduction}

Atrial fibrillation (AF) is the most common arrhythmia, accounting for $1 \%$ to $2 \%$ of the general population, and it will be higher in later years. It is expected that by 2050, the total number of patients with AF worldwide will increase by 2.5 times. There are many common risk factors for atrial fibrillation and coronary heart disease, and both diseases often coexist in the clinic. $10 \%$ to $15 \%$ of patients with coronary heart disease have atrial fibrillation, and the incidence of atrial fibrillation after percutaneous coronary intervention (PCI) is $5 \%$ to $7 \%$. Due to the different mechanisms of atrial fibrillation thrombosis and coronary thrombosis, different antithrombotic methods are needed. How to choose the best antithrombotic (anticoagulant, antiplatelet) treatment program can not only prevent stroke, avoid in-stent restenosis or coronary lesions, but also reduce bleeding risk as much as possible. For patients with coronary artery disease and atrial fibrillation, there are currently five treatment options, including: 1 aspirin antiplatelet therapy alone; 2 dual antiplatelet therapy, aspirin combined with clopidogrel; 3 oral antibiotic alone Coagulation (OAC) therapy; 4 Aspirin or clopidogrel combined with OAC; 5 Triple antithrombotic therapy, aspirin, clopidogrel, and warfarin combined. The optimal antithrombotic treatment for patients with coronary heart disease and atrial fibrillation has been a hot topic in clinical research. Some clinical studies have shown that aspirin has limited antithrombotic effects in atrial fibrillation, and there is no significant difference in the risk of major bleeding or intracranial hemorrhage compared with anticoagulative effects. How to choose antithrombotic therapy in patients with atrial fibrillation and coronary heart disease, and to minimize the bleeding risk while obtaining the maximum antithrombotic benefit, is the key to the development of coronary heart disease with atrial fibrillation anticoagulant therapy program.

\section{Thrombotic Risk Assessment and Bleeding Risk Assessment}

Thrombosis, which is the formation of small pieces of blood flow through the surface of the cardiovascular system, where the surface of the blood vessel is flaking or repaired. In a variable 
fluid-dependent type, the composition of the thrombus includes insoluble fibrin, deposited platelets, accumulated white blood cells, and trapped red blood cells [1]. In the Atrial Fibrillation Guidelines jointly developed by the American Heart Association (AHA), the American College of Cardiology (ACC), and the American Heart Rhythm Association (HRS) in 2014, the CHA2DS2-VASc assessment program expanded the functionality of CHADS2 using the CHA2DS2-VASc scoring method. As a method for assessing the risk of stroke in patients with non-valvular atrial fibrillation, it has been demonstrated to be effective in the clinic [2]. Namely: congestive heart failure / left cardiac insufficiency, hypertension, diabetes, vascular disease, female, age 65-75 years are counted 1 point; age is more than 75 years, stroke / TIA / thrombus history are recorded 2 points; for points For values of $\geq 2$ points, oral anticoagulants such as warfarin are recommended; for patients with a score of 1 , warfarin or aspirin $75-325 \mathrm{mg} / \mathrm{d}$ is recommended; warfarin should be given priority; For the 0 point, it is recommended to use aspirin $75-325 \mathrm{mg} / \mathrm{d}$ or no treatment, giving priority to no treatment.

\section{Atrial Fibrillation Combined with Acute Coronary Syndrome Antithrombotic Therapy}

Acute coronary syndrome (ACS) belongs to the clinical syndrome of coronary atherosclerotic plaque rupture and invasion followed by complete or incomplete occlusive thrombosis, mainly including acute ST-segment elevation myocardial infarction, acute non-ST Segment elevated myocardial infarction, unstable angina (UA). ACS is a common serious cardiovascular disease. As a serious type of coronary heart disease, ACS poses a serious threat to the health of patients. It usually occurs in elderly men and postmenopausal women and is prominent in smoking, hypertension, diabetes, hyperlipidemia, abdominal obesity, and a family history of premature coronary heart disease. The clinical manifestations of ACS are mainly episodes of chest pain and chest tightness. Severe cases are associated with arrhythmia, heart failure, and even the possibility of sudden death. Clinically, patients with suspected symptoms of acute ischemic chest pain should be immediately given a brake. For those who are not contraindicated with ACS, sublingual nitroglycerin should be given immediately and repeated every 5 minutes. The total amount should not exceed $1.5 \mathrm{mg}$. "Time is myocardium and time is life." For ACS, the use of thrombolysis or interventional therapy (PCI) as soon as possible to open the infarct-related artery can significantly reduce mortality, reduce complications, and improve the patient's prognosis. In the 2014 EHRA/ACCA/EAPCI report of the Arrhythmia Working Group of Patients with Acute Coronary Syndrome, it was suggested that patients with atrial fibrillation and ACS should be treated with varying degrees of intensity in response to patient bleeding, stent type, and embolic risk. Antithrombotic therapy, whether it is a bare stent or a drug-eluting stent, recommends clopidogrel + aspirin + warfarin (triple therapy) antiplatelet therapy within 6 months, with a controlled INR of 2.0-2.5. For patients older than 6 months and less than 12 months, clopidogrel plus warfarin is recommended to control the INR between 2.5 and 3.0. It is also emphasized that this treatment is more suitable for those who are at lower risk of bleeding. In the treatment of hyperventilation risk, interventional therapy should be the first choice for RMS, and antithrombotic therapy recommendations are consistent with those with low and medium bleeding risk. In the 9th edition of the Guidelines for Antithrombotic Therapy and Prevention of Thrombosis (ACCP-9) published by the American College of Thoracic and Cardiology Association, based on the 8th edition of the guideline, combined with the latest evidence from evidence-based medicine[2], antithrombotic therapy was performed. Comprehensive and detailed recommendation. For the primary prevention of cardiovascular disease, the use of low-dose aspirin $75-100 \mathrm{mg} / \mathrm{d}$ is better than no use in patients with age $>50$ years and accompanied by cardiovascular disease symptoms; for patients with atrial fibrillation and ACS, and the patients have CHADS2 points For those with a score of 0 , it is recommended that the drug regimen be used for dual antiplatelet therapy for a period of 12 months and then gradually changed to warfarin; for those with a score greater than 1 point, aspirin plus warfarin should be used for a period of 12 months. Then changed to warfarin alone.

Stable angina refers to a relatively stable condition over a period of time (WHO defined a stable disease course $\geq 30$ days). The frequency, duration, and mode of remission of coronary heart disease 
such as angina pectoris are relatively fixed. In addition, stability analysis mainly involves Two aspects, namely stable disease and coronary atherosclerotic plaque stability, did not appear unstable factors such as rupture, ulcer, dissection or thrombosis. In the treatment of such patients, it is generally recommended to give a single antiplatelet drug intervention. For patients with stable angina pectoris complicated with atrial fibrillation, HuaFang combined with antiplatelet drugs was used in the past. In relevant evidence-based evidence studies [3], on the basis of warfarin therapy, combined with aspirin therapy will not effectively reduce the incidence of thrombosis, stroke, etc., and may increase bleeding events to some extent. For stable cardiovascular disease, including history of prior stentless implantation and history of acute ischemic events, the use of vitamin $\mathrm{K}$ antagonist alone is recommended, and antiplatelet therapy is not required during this period. Therefore, in the treatment of stable angina with atrial fibrillation, warfarin regimen is not suitable for treatment. It is recommended to use aspirin in combination with clopidogrel antithrombotic therapy. For patients with high risk of bleeding, aspirin or clopidogrel should be used alone; for stable Patients with angina pectoris with a high risk of atrial fibrillation and thrombosis are recommended to take warfarin antithrombotic therapy, while paying attention to the period of INR monitoring.

\section{Research Progress of Novel Oral Anticoagulant Drugs}

In recent years, new oral anticoagulants and antiplatelet drugs have received more and more attention in the application of coronary heart disease with atrial fibrillation. New oral anticoagulants have been recommended as the preferred antithrombotic drugs for atrial fibrillation because of their good antithrombotic effects and low risk of bleeding. Clinical trials of novel antiplatelet agents (prasugrel) in triple antithrombotic therapy have shown that the risk of major bleeding is significantly higher than that of clopidogrel [4], and the currently recommended P2Y12 receptor inhibitor (ticagrelor) is the guideline It has not been confirmed by clinical trials in the context of triple antithrombotic therapy. The PIONEER AF-PCI study [5] was the first randomized, controlled, multicenter clinical trial to compare novel oral anticoagulants with vitamin $\mathrm{K}$ antagonists in patients with PCI undergoing PCI. Shaban treatment strategies and a vitamin K antagonist treatment strategy are used for the safety of patients with paroxysmal, persistent or permanent non-valvular atrial fibrillation and undergoing PCI. Randomly divide 2124 patients into 3 groups. The first group was treated with rivaroxaban (15 mg once daily) + clopidogrel (75 mg once daily) for 12 months, which was equivalent to the double antithrombotic treatment group in the WOEST study; The group was treated with rivaroxaban (2.5 mg twice daily) + clopidogrel (75 mg once daily) + aspirin (75 to 100 mg once daily) for 12 months. ATLAS study; stratified by dual antiplatelet 1, 6, and 12 months; group 3 by conventional treatment with vitamin $\mathrm{K}$ antagonists (INR controlled at 2.0 to 3.0) + clopidogrel (75 Mg, once daily) + aspirin (75 to $100 \mathrm{mg}$ once daily) for 12 months, stratified by double antiplatelet 1, 6, 12 months. After 12 months of follow-up, the incidence of clinically significant bleeding in the primary safety endpoint group 1 was found (16.8\% vs. $26.7 \%$, HR 0.59 , $95 \%$ CI 0.47 to $0.76, \mathrm{P}<0.001)$, group 2 (18.0\% vs. $26.7 \%$, HR 0.63 , 95\% CI 0.50 to $0.80, \mathrm{P}<0$. 001) were compared with group 3 , respectively. All were statistically significant; the incidence of major adverse cardiovascular events (comprehensive cardiovascular-related death, myocardial infarction, or composite stroke death endpoint) was $6.5 \%$, group 2 is 5 . $6 \%$, group 3 is 6 . At $0 \%$, there was no statistically significant difference between the three groups. The results suggest that rivaroxaban + P2Y12 receptor antagonist or rivaroxaban + dual antiplatelet therapy can significantly reduce clinical bleeding events with comparable efficacy compared with traditional triple antithrombotic therapy. This study shows that the new oral anticoagulant drugs have good prospects for antithrombotic therapy in patients with coronary heart disease and atrial fibrillation.

\section{Conclusion}

In the treatment of patients with coronary artery disease and atrial fibrillation, the risk of thromboembolism and the risk of bleeding should be evaluated before treatment. Then, according to 
the actual situation, such as the type of coronary heart disease, the scores of various scales, etc., comprehensive consideration and selection of appropriate antithrombotic treatment plan. In addition to traditional drugs at the current stage, new oral anticoagulants such as apixaban, rivaroxaban, and dabigatran have continued to deepen through clinical research. Some drugs have been proven to be no worse than warfarin, and has achieved encouraging results in reducing the incidence of intracranial hemorrhage. However, there is less evidence for the safety and efficacy of such drugs and antiplatelet combinations. At this stage of the study, only the new anticoagulant drugs combined with antiplatelet drugs can increase the risk of bleeding to a certain extent. However, it is still unclear whether this treatment plan can be adopted for atrial fibrillation with coronary heart disease. It still needs a large number of clinical studies to confirm.

\section{References}

[1] Dobesh PP. Economic burden of venous thromboembolism inhospitalized patients[J]. harmacotherapy, 2009,29(8):943-53.

[2] EINSTEIN-PE Investigators. Oralrivar oxaban for the treatment of symptomatic pulmonary embolism [J].N Engl JMed, 2012, 5(14):1287-97.

[3] Wang Y, Wang C, Chen Z. Rivaroxaban for the treatment of symptomatic deep-vein thrombosis and pulmonary embolism in Chinese patients: a subgroup analysis of the EINSTEIN DVT and PE studies [J]. Thromb J, 2013,16(1):25.

[4] Matsuo H,Prins M,Lensing AW. Shortened length of hospitalstay with rivaroxaban in patients with symptomatic venousthromboembolism in Japan: the J-EINSTEIN pulmonaryembolism and deep vein thrombosis program[J].Curr Med ResOpin,2015,31(6):1057-61.

[5] Prins $\mathrm{MH}$, Lensing AW, Bauersachs R. Oral rivaroxaban versusstandard therapy for the treatment of symptomatic venousthromboembolism: a pooled analysis of the EINSTEIN-DVT and PE randomized studies[J]. Thromb J,2013, 20(1):21. 